\title{
On a graph of monogenic semigroups
}

\author{
KCh Das ${ }^{1}$, Nihat Akgüneş ${ }^{2 *}$ and A Sinan Çevik ${ }^{2}$
}

\author{
"Correspondence: \\ nakgunes@selcuk.edu.tr \\ ${ }^{2}$ Department of Mathematics, \\ Faculty of Science, Selçuk University, \\ Campus, Konya, 42075, Turkey \\ Full list of author information is \\ available at the end of the article
}

\begin{abstract}
Let us consider the finite monogenic semigroup $\mathcal{S}_{M}$ with zero having elements $\left\{x, x^{2}, x^{3}, \ldots, x^{n}\right\}$. There exists an undirected graph $\Gamma\left(\mathcal{S}_{M}\right)$ associated with $\mathcal{S}_{M}$ whose vertices are the non-zero elements $x, x^{2}, x^{3}, \ldots, x^{n}$ and,$f$ or $1 \leq i, j \leq n$, any two distinct vertices $x^{i}$ and $x^{j}$ are adjacent if $i+j>n$.

In this paper, the diameter, girth, maximum and minimum degrees, domination number, chromatic number, clique number, degree sequence, irregularity index and also perfectness of $\Gamma\left(\mathcal{S}_{M}\right)$ have been established. In fact, some of the results obtained in this section are sharper and stricter than the results presented in DeMeyer et al. (Semigroup Forum 65:206-214, 2002). Moreover, the number of triangles for this special graph has been calculated. In the final part of the paper, by considering two (not necessarily different) graphs $\Gamma\left(\mathcal{S}_{M}^{1}\right)$ and $\Gamma\left(\mathcal{S}_{M}^{2}\right)$, we present the spectral properties to the Cartesian product $\Gamma\left(\mathcal{S}_{M}^{1}\right) \square \Gamma\left(\mathcal{S}_{M}^{2}\right)$.

MSC: $05 \mathrm{C} 10 ; 05 \mathrm{C} 12 ; 06 \mathrm{~A} 07 ; 15 \mathrm{~A} 18 ; 15 \mathrm{~A} 36$
\end{abstract}

Keywords: monogenic semigroup; zero-divisor graph; clique number; chromatic number; independence number; domination number; number of triangles; Cartesian product

\section{Introduction and preliminaries}

The history of studying zero-divisor graphs began with commutative rings in the paper [1], and then it continued with commutative and noncommutative rings in some of the joint papers written by Anderson (see, for instance, [2-4]) and some other authors (see, for instance, $[5,6])$. After that DeMeyer et al. and some other authors studied these special graphs of commutative and noncommutative semigroups [7-9]. Since then a very large number of studies have been added in the literature about zero-divisor graphs. It is obvious that the reason for studying this subject is to give a great opportunity to characterize over the algebraic structure that studied on it.

In [7-9], by considering the (commutative) semigroup $S$ with zero, the zero-divisor graph $\Gamma(S)$ is defined as an undirected graph with vertices $Z(S)^{*}=Z(S) \backslash\{0\}$ and for the set of nonzero zero-divisors of $S$, where for distinct $x, y \in Z(S)^{*}$, the vertices $x$ and $y$ are adjacent if and only if $x y=0$. In the light of this definition, one can always get some new varieties of graphs by changing the rule of adjacency of vertices.

In this paper, we mainly consider the finite multiplicative monogenic semigroup (with zero)

$$
\mathcal{S}_{M}=\left\{x, x^{2}, x^{3}, \ldots, x^{n}\right\}
$$

(0) 2013 Das et al.; licensee Springer. This is an Open Access article distributed under the terms of the Creative Commons Attribution License (http://creativecommons.org/licenses/by/2.0), which permits unrestricted use, distribution, and reproduction in any medium, provided the original work is properly cited. 
Then we define an undirected graph (actually, a type of zero-divisor graph) $\Gamma\left(\mathcal{S}_{M}\right)=(V, E)$ associated to $\mathcal{S}_{M}$ as follows. The vertices are the nonzero zero-divisors (in other words, all nonzero elements) of $\mathcal{S}_{M}$, and any two distinct vertices $x^{i}$ and $x^{j}(1 \leq i, j \leq n)$ are adjacent in case of $x^{i} \cdot x^{j}=0$ with the rule $x^{i} \cdot x^{j}=x^{i+j}=0$ if and only if $i+j>n$ (see Figures 1 and 2 below). We write $x^{i} x^{j} \in E\left(\Gamma\left(\mathcal{S}_{M}\right)\right)$ if vertices $x^{i}$ and $x^{j}$ are adjacent. In this paper, we mainly obtain some certain values for the diameter, girth, maximum and minimum degree, chromatic number, clique number, degree sequence, irregularity index and domination number of the graph $\Gamma\left(\mathcal{S}_{M}\right)$. Moreover, the number of triangles for this special graph has been calculated. We also note that by studying this new graph $\Gamma\left(\mathcal{S}_{M}\right)$, we have reached some strict equalities for the bounds given in [8] for some of the properties of commutative semigroups. For example, in here, we get $\operatorname{diam}\left(\Gamma\left(\mathcal{S}_{M}\right)\right)=2$ and $\operatorname{girth}\left(\Gamma\left(\mathcal{S}_{M}\right)\right)=3$ while they were presented by $\leq 3$ and $\leq 4$, respectively, in [8].

\section{Some spectral properties of $\Gamma\left(\mathcal{S}_{M}\right)$}

In this section, by considering the graph $\Gamma\left(\mathcal{S}_{M}\right)$ defined as in the first section, we will mainly deal with the graph properties, namely diameter, girth, maximum and minimum degrees, domination number and finally irregularity index of it. In fact, it is quite well known that most of these properties can be obtained by checking the distance or the total number of vertices in any graph G. So, the methods in the proofs of the results in this section will be followed by this idea.

We first recall that for any simple graph $G$, the distance (length of the shortest path) between two vertices $u, v$ of $G$ is denoted by $d_{G}(u, v)$. Actually, the diameter of $G$ is defined by

$$
\operatorname{diam}(G)=\max \left\{d_{G}(x, y): x \text { and } y \text { are vertices of } G\right\} .
$$

We thus obtain the following result.

Theorem 1 For any monogenic semigroup $\mathcal{S}_{M}$ as given in (1), the diameter of the graph $\Gamma\left(\mathcal{S}_{M}\right)$ is 2 .

Proof It is clear that the vertex $x$ of $\Gamma\left(\mathcal{S}_{M}\right)$ is pendant, and so the diameter can be figured out by considering the distance between this vertex and one of the other vertices in the vertex set. Therefore, $x$ is only connected with the vertex $x^{n}$, and since $x^{n}$ is adjacent to all other vertices $\left(i . e ., x^{n} \cdot x^{i}=0,1 \leq i \leq n\right)$, we finally get $\operatorname{diam}\left(\Gamma\left(\mathcal{S}_{M}\right)\right)=2$, as required.

It is known that the girth of a simple graph $G$ is the length of the shortest cycle contained in $G$. However, if $G$ does not contain any cycle, then the girth of it is assumed to be infinity.

Theorem 2 For any monogenic semigroup $\mathcal{S}_{M}$ as given in (1), the girth of the graph $\Gamma\left(\mathcal{S}_{M}\right)$ is 3 .

Proof By the definition of $\Gamma\left(\mathcal{S}_{M}\right)$, since $x^{n} \cdot x^{n-1}=0, x^{n-1} \cdot x^{2}=0$ and $x^{n} \cdot x^{2}=0$, we then have $x^{n}-x^{n-1}-x^{2}-x^{n}$, which implies the result, as desired.

The degree $\operatorname{deg}_{G}(v)$ of a vertex $v$ of $G$ is the number of vertices adjacent to $v$. Among all degrees, the maximum degree $\Delta(G)$ (or the minimum degree $\delta(G)$ ) of $G$ is the number of 


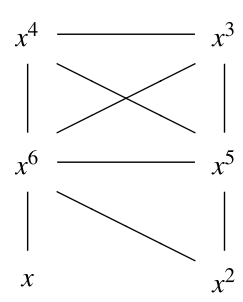

Figure 1 The graph $\Gamma\left(\mathcal{S}_{M_{6}}\right)$.

the largest (or the smallest) degrees in $G$ (see [10]). By considering maximum or minimum degrees, another result can be presented as follows.

Theorem 3 For any monogenic semigroup $\mathcal{S}_{M}$ as given in (1), the maximum and minimum degrees of $\Gamma\left(\mathcal{S}_{M}\right)$ are

$$
\Delta\left(\Gamma\left(\mathcal{S}_{M}\right)\right)=n-1 \quad \text { and } \quad \delta\left(\Gamma\left(\mathcal{S}_{M}\right)\right)=1
$$

Proof Let us consider the vertex $x^{n}$ of $\Gamma\left(\mathcal{S}_{M}\right)$. It is clear that $x^{n} \cdot x^{i}=0$ for any $1 \leq i \leq n$, as $n+i>n$. By the definition of $\Gamma\left(\mathcal{S}_{M}\right)$, we get $x^{n} x^{i} \in E\left(\Gamma\left(\mathcal{S}_{M}\right)\right), 1 \leq i \leq n$. Thus, we have $\Delta\left(\Gamma\left(\mathcal{S}_{M}\right)\right)=n-1$.

On the other hand, let us consider the vertex $x$ of $\Gamma\left(\mathcal{S}_{M}\right)$. Then the equality $x \cdot x^{i}=0$ satisfies only if $i=n$. Nevertheless, for every $i=\{1,2, \ldots, n-1\}$, we have $x \cdot x^{i} \neq 0$. That means the unique vertex $x$ is only connected to the vertex $x^{n}\left(i . e ., x^{n} x \in E\left(\Gamma\left(\mathcal{S}_{M}\right)\right)\right.$ ), which implies $\delta\left(\Gamma\left(\mathcal{S}_{M}\right)\right)=1$, as required.

Example 1 Consider the graph $\Gamma\left(\mathcal{S}_{M_{6}}\right)$, as drawn in Figure 1, with the vertex set $V\left(\Gamma\left(\mathcal{S}_{M_{6}}\right)\right)=\left\{x, x^{2}, x^{3}, x^{4}, x^{5}, x^{6}\right\}$. By Theorems 1,2 and 3 , we certainly have $\operatorname{diam}\left(\Gamma\left(\mathcal{S}_{M_{6}}\right)\right)=$ $2, \operatorname{girth}\left(\Gamma\left(\mathcal{S}_{M_{6}}\right)\right)=3, \Delta\left(\Gamma\left(\mathcal{S}_{M_{6}}\right)\right)=5, \delta\left(\Gamma\left(\mathcal{S}_{M_{6}}\right)\right)=1$.

The degree sequence, denote by $D S(G)$, is a sequence of degrees of vertices of a graph $G$. In [11], a new parameter for graphs, namely the irregularity index of $G$, has been recently defined and denoted by $M W B(G)$. In fact $M W B(G)$ is the number of distinct terms in the set $D S(G)$. (At this point, we should note that although this new index is denoted by $t(G)$ in the paper [11], we prefer to denote it by $M W B(G)$ not to make any confusion with the material in Section 4 of this paper.)

We recall that for a real number $r$, the notation $\lfloor r\rfloor$ denotes the greatest integer $\leq r$ while $\lceil r\rceil$ denotes the least integer $\geq r$. This fact will be needed for some of the theorems in this paper.

Theorem 4 Let $\mathcal{S}_{M}$ be a monogenic semigroup as given in (1). Then the degree sequence and irregularity index of $\Gamma\left(\mathcal{S}_{M}\right)$ are given by

$$
D S\left(\Gamma\left(\mathcal{S}_{M}\right)\right)=\left\{1,2,3, \ldots,\left\lfloor\frac{n}{2}\right\rfloor-1,\left\lfloor\frac{n}{2}\right\rfloor,\left\lfloor\frac{n}{2}\right\rfloor,\left\lfloor\frac{n}{2}\right\rfloor+1,\left\lfloor\frac{n}{2}\right\rfloor+2, \ldots, n-2, n-1\right\}
$$

and $M W B\left(\Gamma\left(\mathcal{S}_{M}\right)\right)=n-1$, respectively. 
Proof In $\Gamma\left(\mathcal{S}_{M}\right)$, since the vertex $x$ is connected only with the vertex $x^{n}$, then we clearly obtain that the degree of $x$ is 1 . Secondly, let us consider the vertex $x^{2} \in V\left(\Gamma\left(\mathcal{S}_{M}\right)\right)$. Then, as a similar idea, it is only connected with the vertices $x^{n}$ and $x^{n-1}$, which implies that the degree of $x^{2}$ is equal to 2 . Now, if we apply the same progress to all remaining vertices, then we see that

- the degree of vertex $x^{\left\lfloor\frac{n}{2}\right\rfloor-1}$ is $\left\lfloor\frac{n}{2}\right\rfloor-1$ and

- the degree of vertex $x^{\left\lfloor\frac{n}{2}\right\rfloor}$ is $\left\lfloor\frac{n}{2}\right\rfloor$, but

- the vertex $x^{\left\lfloor\frac{n}{2}\right\rfloor+1}$ has the same degree as the vertex $x^{\left\lfloor\frac{n}{2}\right\rfloor}$.

Moreover,

- the degree of vertex $x^{\left\lfloor\frac{n}{2}\right\rfloor+2}$ is $\left\lfloor\frac{n}{2}\right\rfloor+1$ and

- the degree of vertex $x^{\left\lfloor\frac{n}{2}\right\rfloor+3}$ is $\left\lfloor\frac{n}{2}\right\rfloor+2$.

Now, if we keep following the same procedure, then we get that

- the degree of vertex $x^{n-1}$ is $n-2$, while the degree of vertex $x^{n}$ is $n-1$.

Hence, by the definition of degree sequence, we clearly obtain the set $D S\left(\Gamma\left(\mathcal{S}_{M}\right)\right)$ as depicted in the theorem. Nevertheless, it is easily seen that the irregularity index $\operatorname{MWB}\left(\Gamma\left(\mathcal{S}_{M}\right)\right)=n-1$, as required.

A subset $D$ of the vertex set $V(G)$ of a graph $G$ is called a dominating set if every vertex $V(G) \backslash D$ is joined to at least one vertex of $D$ by an edge. Additionally, the domination number $\gamma(G)$ is the number of vertices in the smallest dominating set for $G$ (see [10]).

Now, in our case, by considering the definition of $\Gamma\left(\mathcal{S}_{M}\right)$, the vertex $x^{n}$ is the only element adjacent to all the other vertices. In other words, $x^{n} x^{i} \in E\left(\Gamma\left(\mathcal{S}_{M}\right)\right)$ for $1 \leq i \leq n$, and so the dominating set contains only the element $x^{n}$. This simple fact gives the following result about the domination number of $\Gamma\left(\mathcal{S}_{M}\right)$.

Theorem 5 Let $\mathcal{S}_{M}$ be a monogenic semigroup as given in (1). Then

$$
\gamma\left(\Gamma\left(\mathcal{S}_{M}\right)\right)=1
$$

Example 2 As an example of Theorems 4 and 5, let us consider the graphs $\Gamma\left(\mathcal{S}_{M_{4}}\right)$ and $\Gamma\left(\mathcal{S}_{M_{5}}\right)$ as drawn in Figure 2. In here, $\gamma\left(\Gamma\left(\mathcal{S}_{M_{4}}\right)\right)=1, D S\left(\Gamma\left(\mathcal{S}_{M_{4}}\right)\right)=\{1,2,2,3\}$ and $\operatorname{MWB}\left(\Gamma\left(\mathcal{S}_{M_{4}}\right)\right)=3$. Moreover, $\operatorname{DS}\left(\Gamma\left(\mathcal{S}_{M_{5}}\right)\right)=\{1,2,2,3,4\}$ and $M W B\left(\mathcal{S}_{M_{5}}\right)=4$.

We note that for the graph $\Gamma\left(\mathcal{S}_{M_{6}}\right)$ in Example 1, $M W B\left(\Gamma\left(\mathcal{S}_{M_{6}}\right)\right)=5$.

\section{Perfectness property of $\Gamma\left(\mathcal{S}_{M}\right)$}

Since perfect graphs are directly related to the terms coloring and clique numbers, let us start this section by reminding the definitions of them.

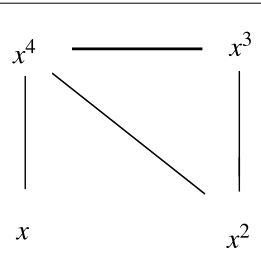

(i)

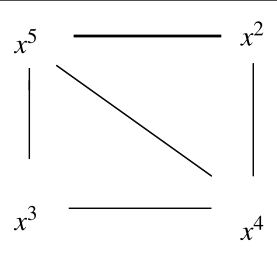

(ii)

Figure 2 Graphs $\Gamma\left(\mathcal{S}_{M_{4}}\right)$ and $\Gamma\left(\mathcal{S}_{M_{5}}\right)$. 
Basically, the coloring of $G$ is to be an assignment of colors (elements of some set) to the vertices of $G$, one color to each vertex, so that adjacent vertices are assigned distinct colors. If $n$ colors are used, then the coloring is referred to as $n$-coloring. If there exists an $n$-coloring of $G$, then $G$ is called $n$-colorable. The minimum number $n$ for which $G$ is $n$-colorable is called the chromatic number of $G$ and is denoted by $\chi(G)$. In addition, there exists another graph parameter, namely the clique of a graph $G$. In fact, depending on the vertices, each of the maximal complete subgraphs of $G$ is called a clique. Moreover, the largest number of vertices in any clique of $G$ is called the clique number and denoted by $\omega(G)$. In general, it is well known that $\chi(G) \geq \omega(G)$ for any graph $G$ (see, for instance, [10]). For every induced subgraph $H \subseteq G$ of $G$, if $\chi(H)=\omega(H)$, then $G$ is called a perfect graph [12].

In here, we will state and prove the chromatic and clique numbers for the graph $\Gamma\left(\mathcal{S}_{M}\right)$ separately, and so the perfectness of this special graph will be obtained.

Theorem 6 The chromatic number of $\Gamma\left(\mathcal{S}_{M}\right)$ is equal to

$$
\chi\left(\Gamma\left(\mathcal{S}_{M}\right)\right)=1+\left\lceil\frac{n-1}{2}\right\rceil .
$$

Proof As usual, let us consider the graph $\Gamma\left(\mathcal{S}_{M}\right)$ associated with $\mathcal{S}_{M}$ as defined in (1). Now, if we first take account of the vertex $x^{n}$, then it is easy to see that $x^{n}$ is adjacent to all the other vertices. That means the color used for $x^{n}$ cannot be used for the remaining vertices. So, let us suppose that the color for $x^{n}$ is labeled $\mathcal{C}_{1}$.

Secondly, let us consider the vertex $x^{n-1}$. Since $x^{n-1}$ is adjacent to all vertices except the vertex $x$, the color for $x^{n-1}$, say $\mathcal{C}_{2}$, can also be used only for $x$. Similarly, the vertex $x^{n-2}$ is adjacent to all vertices except the vertices $x$ and $x^{2}$. Thus the color, say $\mathcal{C}_{3}$, for $x^{n-2}$ can also be used only for the vertex $x^{2}$. (The color $\mathcal{C}_{2}$ has already been used for $x$ in the previous step.)

By applying same progress, one can see that to handle the number of coloring for all vertices in the set $V\left(\Gamma\left(\mathcal{S}_{M}\right)\right)$, we must add 1 to the least integer $\geq \frac{n-1}{2}$. In other words, a total $1+\left\lceil\frac{n-1}{2}\right\rceil$ colors should be needed, which gives the required chromatic number in the theorem.

The following lemma (proof can be seen directly by mathematical induction) plays a central role in the proof of Theorem 7 below.

Lemma 1 For any $n \in \mathbb{N}^{+}$, there always exists $n-\left\lceil\frac{n}{2}\right\rceil=\left\lceil\frac{n-1}{2}\right\rceil$.

Theorem 7 The clique number of $\Gamma\left(\mathcal{S}_{M}\right)$ is equal to

$$
\omega\left(\Gamma\left(\mathcal{S}_{M}\right)\right)=1+\left\lceil\frac{n-1}{2}\right\rceil .
$$

Proof Now, let us consider the complete subgraph $A \subseteq \Gamma\left(\mathcal{S}_{M}\right)$. For all distinct vertices $x^{i}, x^{j} \in V(A)$, we have

$$
x^{i} \cdot x^{j}=x^{i+j}=0, \quad \text { i.e., } x^{i} x^{j} \in E\left(\Gamma\left(\mathcal{S}_{M}\right)\right) \text { for all } i \text { and } j .
$$


In fact, (2) can be satisfied only in the case if the sum $i+j$ would be at least equal to the $n+1$. Therefore, for any two vertices $x^{i}$ and $x^{j}$, the values of $i$ and $j$ must be at least

$$
i=\left\lceil\frac{n}{2}\right\rceil \text { and } j=\left\lceil\frac{n}{2}\right\rceil+1
$$

as $\left\lceil\frac{n}{2}\right\rceil+\left\lceil\frac{n}{2}\right\rceil+1 \geq n+1$. This process will give us that $A \subseteq \Gamma\left(\mathcal{S}_{M}\right)$ is a complete subgraph with the vertex set

$$
V(A)=\left\{x^{\left\lceil\frac{n}{2}\right\rceil}, x^{\left\lceil\frac{n}{2}\right\rceil+1}, \ldots, x^{n-1}, x^{n}\right\}
$$

It is easy to see that the number of elements in $V(A)$ is $n-\left\lceil\frac{n}{2}\right\rceil+1$, which equals $\left\lceil\frac{n-1}{2}\right\rceil+1$ by Lemma 1.

By contradiction, we will show that the set $V(A)$ is maximal. Suppose to the contrary that $|V(A)|>\left\lceil\frac{n-1}{2}\right\rceil+1$. If any two vertices $x^{i}$ and $x^{j}\left(x^{i}, x^{j} \in V\left(\Gamma\left(\mathcal{S}_{M}\right)\right), 1 \leq i, j \leq\left\lceil\frac{n}{2}\right\rceil-1\right)$ is in $V(A)$, then we arrive at a contradiction, as $i+j \leq n-1$. Otherwise, exactly any one vertex $x^{i}\left(x^{i} \in V\left(\Gamma\left(\mathcal{S}_{M}\right)\right), 1 \leq i \leq\left\lceil\frac{n}{2}\right\rceil-1\right)$ is in $V(A)$ as $|V(A)|>\left\lceil\frac{n-1}{2}\right\rceil+1$. Again, we arrive at a contradiction as $i+\left\lceil\frac{n}{2}\right\rceil \leq n, 1 \leq i \leq\left\lceil\frac{n}{2}\right\rceil-1$. Thus, the set $V(A)$ is maximal. Hence, $\omega\left(\Gamma\left(\mathcal{S}_{M}\right)\right)=1+\left\lceil\frac{n-1}{2}\right\rceil$, as required.

Now, by keeping in our mind the definition of perfect graphs [12] as depicted in the beginning of this section and considering Theorems 6, 7, we can obtain the perfectness of the graph $\Gamma\left(\mathcal{S}_{M}\right)$ as in the following corollary.

Remark 1 Since $\chi\left(\Gamma\left(\mathcal{S}_{M}\right)\right)=\omega\left(\Gamma\left(\mathcal{S}_{M}\right)\right)=1+\left\lceil\frac{n-1}{2}\right\rceil$, the graph $\Gamma\left(\mathcal{S}_{M}\right)$ is perfect.

Notice that for $\Gamma\left(\mathcal{S}_{M_{5}}\right)$ and $\Gamma\left(\mathcal{S}_{M_{6}}\right)$ as drawn in Figures 1 and 2(ii), respectively, we have $\chi\left(\Gamma\left(\mathcal{S}_{M_{5}}\right)\right)=3=\omega\left(\Gamma\left(\mathcal{S}_{M_{5}}\right)\right)$ and $\chi\left(\Gamma\left(\mathcal{S}_{M_{6}}\right)\right)=4=\omega\left(\Gamma\left(\mathcal{S}_{M_{6}}\right)\right)$, respectively.

We recall that any graph $G$ is called Berge if no induced subgraph of $G$ is an odd cycle of length of at least five or the complement of one (see [13]). The following lemma proved by Chudnovsky et al. in [14] figures out the relationship between perfect and Berge graphs. (This lemma is named strong perfect conjecture in some studies.)

Lemma 2 ([14]) A graph is perfect if and only if it is Berge.

By using this relationship depicted in Lemma 2, one can also prove the perfectness of $\Gamma\left(\mathcal{S}_{M}\right)$ as in the following result.

Theorem 8 Let $\mathcal{S}_{M}$ be a monogenic semigroup as in (1). Then the graph of $\Gamma\left(\mathcal{S}_{M}\right)$ is perfect.

Proof Assume that any induced subgraph of $\Gamma\left(\mathcal{S}_{M}\right)$ contains an odd cycle $C_{2 k+1}$ and its number of vertices are $2 k+1(k \geq 2)$. Let us assume that these vertices are $x^{a_{1}}, x^{a_{2}}, \ldots, x^{a_{2 k+1}}$ in $C_{2 k+1}$ such that $x^{a_{1}}-x^{a_{2}}-x^{a_{3}}-\cdots-x^{a_{2 k+1}}-x^{a_{1}}$, where $a_{1}<a_{2}<\cdots<a_{2 k+1}$. Since $x^{a_{i}} x^{a_{i+1}} \in$ $E\left(\Gamma\left(\mathcal{S}_{M}\right)\right)(1 \leq i \leq 2 k)$ and $x^{a_{1}} x^{a_{2 k+1}} \in E\left(\Gamma\left(\mathcal{S}_{M}\right)\right)$, by the definition of $\Gamma\left(\mathcal{S}_{M}\right)$, we get $x^{a_{1}} x^{a_{j}} \in$ $E\left(\Gamma\left(\mathcal{S}_{M}\right)\right), 3 \leq j \leq 2 k$ as $a_{1}+a_{j}>a_{1}+a_{2} \geq n+1$. This implies that no odd cycle induced subgraph of length of at least 5 is in $\Gamma\left(\mathcal{S}_{M}\right)$, which is a contradiction. Finally, by Lemma 2, we conclude that $\Gamma\left(\mathcal{S}_{M}\right)$ is perfect. 
Moreover, by [12], since the complement of a perfect graph is also perfect, we also obtain that the complement $\overline{\Gamma\left(\mathcal{S}_{M}\right)}$ of $\Gamma\left(\mathcal{S}_{M}\right)$ is perfect. As a final note, we may refer to $[10,15]$ for some other properties of perfect graphs which are clearly satisfied for $\Gamma\left(\mathcal{S}_{M}\right)$.

\section{Number of triangles in $\Gamma\left(\mathcal{S}_{M}\right)$}

In this section, by using the spectral graph theory, we count the number of triangles in the graph $\Gamma\left(\mathcal{S}_{M}\right)$ associated with $\mathcal{S}_{M}$ as defined in (1).

We recall that the notation $t\left(\Gamma\left(\mathcal{S}_{M}\right)\right)$ denotes the number of triangles for any simple undirected graph $\Gamma\left(\mathcal{S}_{M}\right)$. In fact, the following lemma on $t\left(\Gamma\left(\mathcal{S}_{M}\right)\right)$ will be needed for our main result of this section.

Lemma 3 ([16]) Let $\Gamma\left(\mathcal{S}_{M}\right)$ be a simple graph of order $n$, and let $x^{i}, x^{j} \in V\left(\Gamma\left(\mathcal{S}_{M}\right)\right)$. Suppose that $N_{i}$ and $N_{j}$ are the neighbor sets of $x^{i}$ and $x^{j}$, respectively. Then

$$
\sum_{x^{i} x_{j} \in E\left(\Gamma\left(\mathcal{S}_{M}\right)\right)}\left|N_{i} \cap N_{j}\right|=3 t\left(\Gamma\left(\mathcal{S}_{M}\right)\right)
$$

where $\left|N_{i} \cap N_{j}\right|$ denotes the cardinality of common neighbors.

Before presenting this result, let us consider the adjacency matrix (here the first row and column correspond to vertex $x^{n}$, the second row and column correspond to vertex $x^{n-1}, \ldots$, etc.) of $\Gamma\left(\mathcal{S}_{M}\right)$ which is defined by its entries $a_{i j}=1$ if $x^{i} x^{j} \in E\left(\Gamma\left(\mathcal{S}_{M}\right)\right)$ and 0 otherwise (see $A\left(\Gamma\left(\mathcal{S}_{M}\right)\right)$ in Figure 3). Since $A\left(\Gamma\left(\mathcal{S}_{M}\right)\right)$ is symmetric, its eigenvalues are real. Without loss of generality, we can write them as $\lambda_{1} \geq \lambda_{2} \geq \cdots \geq \lambda_{n}$ and call them the eigenvalues of $\Gamma\left(\mathcal{S}_{M}\right)$. Moreover, the second power $A^{2}\left(\Gamma\left(\mathcal{S}_{M}\right)\right)$ of the adjacency matrix is written as the form given in Figure 4.

Theorem 9 Let $\Gamma\left(\mathcal{S}_{M}\right)$ be the graph of order $n$ associated with $\mathcal{S}_{M}$ as defined in (1). Also, let $t\left(\Gamma\left(\mathcal{S}_{M}\right)\right)$ be the number of triangles in $\Gamma\left(\mathcal{S}_{M}\right)$. Then

$$
t\left(\Gamma\left(\mathcal{S}_{M}\right)\right)= \begin{cases}\frac{n(n-1)(n-2)}{24} & \text { when } n \text { is even } \\ \frac{(n-3)\left(n^{2}-1\right)}{24} & \text { when } n \text { is odd }\end{cases}
$$

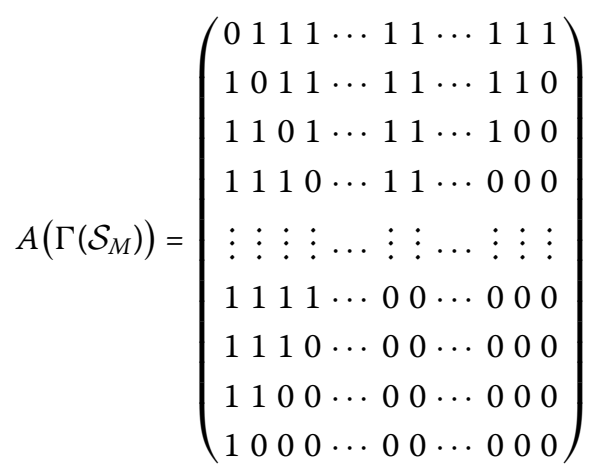

Figure 3 The matrix $A\left(\Gamma\left(\mathcal{S}_{M}\right)\right)$. 


$$
\left(\begin{array}{cccccccccccc}
n-1 & n-3 & n-4 & n-5 & \cdots & \left\lfloor\frac{n}{2}\right\rfloor-1 & \left\lfloor\frac{n}{2}\right\rfloor-1 & \cdots & 2 & 1 & 0 \\
n-3 & n-2 & n-4 & n-5 & \cdots & \left\lfloor\frac{n}{2}\right\rfloor-1 & \left\lfloor\frac{n}{2}\right\rfloor-1 & \cdots & 2 & 1 & 1 \\
n-4 & n-4 & n-3 & n-5 & \cdots & \left\lfloor\frac{n}{2}\right\rfloor-1 & \left\lfloor\frac{n}{2}\right\rfloor-1 & \cdots & 2 & 2 & 1 \\
n-5 & n-5 & n-5 & n-4 & \cdots & \left\lfloor\frac{n}{2}\right\rfloor-1 & \left\lfloor\frac{n}{2}\right\rfloor-1 & \cdots & 3 & 2 & 1 \\
\vdots & \vdots & \vdots & \vdots & \cdots & \vdots & \vdots & \cdots & \vdots & \vdots & \vdots \\
\left\lfloor\frac{n}{2}\right\rfloor-1 & \left\lfloor\frac{n}{2}\right\rfloor-1 & \left\lfloor\frac{n}{2}\right\rfloor-1 & \left\lfloor\frac{n}{2}\right\rfloor-1 & \cdots & \left\lfloor\frac{n}{2}\right\rfloor & \left\lfloor\frac{n}{2}\right\rfloor-1 & \cdots & 3 & 2 & 1 \\
\left\lfloor\frac{n}{2}\right\rfloor-1 & \left\lfloor\frac{n}{2}\right\rfloor-1 & \left\lfloor\frac{n}{2}\right\rfloor-1 & \left\lfloor\frac{n}{2}\right\rfloor-1 & \cdots & \left\lfloor\frac{n}{2}\right\rfloor-1 & \left\lfloor\frac{n}{2}\right\rfloor & \cdots & 3 & 2 & 1 \\
\vdots & \vdots & \vdots & \vdots & \cdots & \vdots & \vdots & \cdots & \vdots & \vdots \\
3 & 3 & 3 & 3 & \cdots & 4 & 4 & \cdots & 3 & 2 & 1 \\
2 & 2 & 2 & 3 & \cdots & 3 & 3 & \cdots & 3 & 2 & 1 \\
1 & 1 & 2 & 2 & \cdots & 2 & 2 & \cdots & 2 & 2 & 1 \\
0 & 1 & 1 & 1 & \cdots & 1 & 1 & \cdots & 1 & 1 & 1
\end{array}\right) .
$$

Figure 4 The matrix $A^{2}\left(\Gamma\left(\mathcal{S}_{M}\right)\right)$.

Proof It is well known that for any graph $\Gamma\left(\mathcal{S}_{M}\right)$, we have

$$
\sum_{i=1}^{n} \lambda_{i}=0, \quad \sum_{i=1}^{n} \lambda_{i}^{2}=\sum_{i=1}^{n} d_{i}=2 m, \quad m \text { is the number of edges in } \Gamma\left(\mathcal{S}_{M}\right)
$$

and

$$
\begin{aligned}
\sum_{i=1}^{n} \lambda_{i}^{3} & =\sum_{i=1}^{n} \sum_{v_{j}: v_{i} v_{j} \in E\left(\Gamma\left(\mathcal{S}_{M}\right)\right)}\left|N_{i} \cap N_{j}\right|=2 \sum_{v_{i} v_{j} \in E\left(\Gamma\left(\mathcal{S}_{M}\right)\right)}\left|N_{i} \cap N_{j}\right| \\
& =6 t\left(\Gamma\left(\mathcal{S}_{M}\right)\right) \quad \text { by Lemma } 3 .
\end{aligned}
$$

We now consider $A^{2}\left(\Gamma\left(\mathcal{S}_{M}\right)\right)=\left(b_{i, j}\right)_{n \times n}$ and

$$
A^{3}\left(\Gamma\left(\mathcal{S}_{M}\right)\right)=A\left(\Gamma\left(\mathcal{S}_{M}\right)\right) \cdot A^{2}\left(\Gamma\left(\mathcal{S}_{M}\right)\right)=\left(c_{i, j}\right)_{n \times n}
$$

From the above, we certainly have

$$
\begin{aligned}
c_{1,1}= & b_{1,2}+b_{1,3}+b_{1,4}+\cdots+b_{1,\left\lceil\frac{n}{2}\right\rceil-1}+b_{1,\left\lceil\frac{n}{2}\right\rceil}+b_{1,\left\lceil\frac{n}{2}\right\rceil+1}+b_{1,\left\lceil\frac{n}{2}\right\rceil+2}+\cdots+b_{1, n-2}+b_{1, n-1} \\
= & (n-3)+(n-4)+(n-5)+\cdots+\left\lfloor\frac{n}{2}\right\rfloor+\left(\left\lfloor\frac{n}{2}\right\rfloor-1\right)+\left(\left\lfloor\frac{n}{2}\right\rfloor-1\right) \\
& +\left(\left\lfloor\frac{n}{2}\right\rfloor-2\right)+\cdots+3+2+1 \\
= & \frac{(n-3)(n-2)}{2}+\left(\left\lfloor\frac{n}{2}\right\rfloor-1\right), \\
c_{2,2}= & b_{2,1}+b_{2,3}+b_{2,4}+\cdots+b_{2,\left\lceil\frac{n}{2}\right\rceil-1}+b_{2,\left\lceil\frac{n}{2}\right\rceil}+b_{2,\left\lceil\frac{n}{2}\right\rceil+1} \\
& +b_{2,\left\lceil\frac{n}{2}\right\rceil+2}+\cdots+b_{2, n-3}+b_{2, n-2}+b_{2, n-1}
\end{aligned}
$$




$$
\begin{aligned}
= & (n-3)+(n-4)+(n-5)+\cdots+\left\lfloor\frac{n}{2}\right\rfloor+\left(\left\lfloor\frac{n}{2}\right\rfloor-1\right) \\
& +\left(\left\lfloor\frac{n}{2}\right\rfloor-1\right)+\left(\left\lfloor\frac{n}{2}\right\rfloor-2\right)+\cdots+3+2+1 \\
= & \frac{(n-3)(n-2)}{2}+\left(\left\lfloor\frac{n}{2}\right\rfloor-1\right), \\
c_{3,3}= & b_{3,1}+b_{3,2}+b_{3,4}+\cdots+b_{3,\left\lceil\frac{n}{2}\right\rceil-1}+b_{3,\left\lceil\frac{n}{2}\right\rceil}+b_{3,\left\lceil\frac{n}{2}\right\rceil+1} \\
& +b_{3,\left\lceil\frac{n}{2}\right\rceil+2}+\cdots+b_{3, n-4}+b_{3, n-3}+b_{3, n-2} \\
= & (n-4)+(n-4)+(n-5)+\cdots+\left\lfloor\frac{n}{2}\right\rfloor+\left(\left\lfloor\frac{n}{2}\right\rfloor-1\right) \\
& +\left(\left\lfloor\frac{n}{2}\right\rfloor-1\right)+\left(\left\lfloor\frac{n}{2}\right\rfloor-2\right)+\cdots+4+3+2 \\
= & \frac{(n-3)(n-2)}{2}+\left(\left\lfloor\frac{n}{2}\right\rfloor-1\right)-2
\end{aligned}
$$

and

$$
\begin{aligned}
c_{4,4}= & b_{4,1}+b_{4,2}+b_{4,3}+\cdots+b_{4,\left\lceil\frac{n}{2}\right\rceil-1}+b_{4,\left\lceil\frac{n}{2}\right\rceil}+b_{4,\left\lceil\frac{n}{2}\right\rceil+1} \\
& +b_{4,\left\lceil\frac{n}{2}\right\rceil+2}+\cdots+b_{4, n-5}+b_{4, n-4}+b_{4, n-3} \\
= & (n-5)+(n-5)+(n-5)+\cdots+\left\lfloor\frac{n}{2}\right\rfloor+\left(\left\lfloor\frac{n}{2}\right\rfloor-1\right) \\
& +\left(\left\lfloor\frac{n}{2}\right\rfloor-1\right)+\left(\left\lfloor\frac{n}{2}\right\rfloor-2\right)+\cdots+5+4+3 \\
= & \frac{(n-3)(n-2)}{2}+\left(\left\lfloor\frac{n}{2}\right\rfloor-1\right)-6 .
\end{aligned}
$$

By iterating this above progress, we get

$$
c_{\left\lceil\frac{n}{2}\right\rceil,\left\lceil\frac{n}{2}\right\rceil}=\left(\left\lfloor\frac{n}{2}\right\rfloor-1\right)+\left(\left\lfloor\frac{n}{2}\right\rfloor-1\right)+\left(\left\lfloor\frac{n}{2}\right\rfloor-1\right)+\cdots+\left(\left\lfloor\frac{n}{2}\right\rfloor-1\right),
$$

which actually equals

$$
\left(\left\lfloor\frac{n}{2}\right\rfloor-1\right)\left\lfloor\frac{n}{2}\right\rfloor
$$

Similarly, we obtain

$$
c_{\left\lceil\frac{n}{2}\right\rceil+1,\left\lceil\frac{n}{2}\right\rceil+1}=\left(\left\lfloor\frac{n}{2}\right\rfloor-1\right)\left\lfloor\frac{n}{2}\right\rfloor .
$$

By continuing these calculations, we finally reach

$$
\begin{aligned}
& c_{n-3, n-3}=3+3+3+3=12, \quad c_{n-2, n-2}=2+2+2=6, \\
& c_{n-1, n-1}=1+1=2, \quad c_{n, n}=0 .
\end{aligned}
$$

We have two possible cases for $n$ : 
- If $n$ is even, then

$$
\begin{aligned}
\sum_{i=1}^{n} c_{i, i} & =\left[\frac{(n-3)(n-2)}{2}+\frac{n-2}{2}\right] \times \frac{n}{2}+\frac{n-2}{2} \times \frac{n}{2} \\
& =\frac{n(n-2)^{2}}{4}+\frac{n(n-2)}{4}=\frac{n(n-1)(n-2)}{4} .
\end{aligned}
$$

- If $n$ is odd, then

$$
\begin{aligned}
\sum_{i=1}^{n} c_{i, i} & =\left[\frac{(n-3)(n-2)}{2}+\frac{n-3}{2}\right] \times \frac{n+1}{2} \\
& =\frac{(n-1)(n-3)}{2} \times \frac{n+1}{2}=\frac{(n-3)\left(n^{2}-1\right)}{4} .
\end{aligned}
$$

Since the eigenvalues of the matrix $A^{3}\left(\Gamma\left(\mathcal{S}_{M}\right)\right)$ are $\lambda_{1}^{3}, \lambda_{2}^{3}, \ldots, \lambda_{n}^{3}$, by considering (4), we get

$$
6 t\left(\Gamma\left(\mathcal{S}_{M}\right)\right)=\sum_{i=1}^{n} \lambda_{i}^{3}=\operatorname{tr}\left(A^{3}\left(\Gamma\left(\mathcal{S}_{M}\right)\right)\right)=\sum_{i=1}^{n} c_{i, i}
$$

Using (5) and (6) in $t\left(\Gamma\left(\mathcal{S}_{M}\right)\right)=\frac{1}{6} \sum_{i=1}^{n} c_{i, i}$, we get the result, as desired.

By Figures 1 and 2, it is quite easy to see that $t\left(\Gamma\left(\mathcal{S}_{M_{4}}\right)\right), t\left(\Gamma\left(\mathcal{S}_{M_{5}}\right)\right)$ and $t\left(\Gamma\left(\mathcal{S}_{M_{6}}\right)\right)$ are equal to 1,2 and 5 , respectively.

\section{The Cartesian product of $\Gamma\left(\mathcal{S}_{M}^{1}\right)$ and $\Gamma\left(\mathcal{S}_{M}^{2}\right)$}

For given arbitrary graphs $G_{1}$ and $G_{2}$, the Cartesian product $G_{1} \square G_{2}$ is defined as the graph on the vertex set $V\left(G_{1}\right) \times V\left(G_{2}\right)$ with vertices $u=\left(u_{1}, u_{2}\right)$ and $v=\left(v_{1}, v_{2}\right)$ which are connected by an edge if and only if either $u_{1}=v_{1}$ and $u_{2} v_{2} \in E\left(G_{2}\right)$ or $u_{2}=v_{2}$ and $u_{1} v_{1} \in$ $E\left(G_{1}\right)$. This subject has been studied extensively by several authors (see, for instance, [1719]).

Throughout this section, we will assume that $\mathcal{S}_{M}^{1}$ and $\mathcal{S}_{M}^{2}$ denote monogenic semigroups with 0 defined by the sets

$$
\left\{x_{1}, x_{1}^{2}, x_{1}^{3}, \ldots, x_{1}^{n}\right\} \text { and }\left\{x_{2}, x_{2}^{2}, x_{2}^{3}, \ldots, x_{2}^{m}\right\}
$$

respectively, as given in (1). Without loss of generality, we can assume that $n \geq m$. In this section, the following results will be dealt with: the diameter, girth, chromatic number and clique number of the graph $\Gamma\left(\mathcal{S}_{M}^{1}\right) \square \Gamma\left(\mathcal{S}_{M}^{2}\right)$.

For simplicity, the graph $\Gamma\left(\mathcal{S}_{M}^{1}\right) \square \Gamma\left(\mathcal{S}_{M}^{2}\right)$ will be denoted by a single letter $\mathcal{T}$.

Theorem 10 For any two monogenic semigroups $\mathcal{S}_{M}^{1}$ and $\mathcal{S}_{M}^{2}$ as defined in (7),

$$
\operatorname{diam}(\mathcal{T})=4
$$

Proof Since the vertex $\left(x_{1}, x_{2}\right)$ of the graph $\mathcal{T}$ definitely has neighborhoods, the diameter can be figured out by considering the distance between $\left(x_{1}, x_{2}\right)$ and one of the other vertices in $V(\mathcal{T})$. It is easily deduced that $\left(x_{1}, x_{2}\right)$ is just adjacent to the vertices $\left(x_{1}, x_{2}^{m}\right)$ and $\left(x_{1}^{n}, x_{2}\right)$. 
However, for $1 \leq i<n$ and $1 \leq j<m$, since $x_{1}^{n} \cdot x_{1}^{i}=0$ and $x_{2}^{j} \cdot x_{2}^{m}=0$, we clearly obtain each of $\left(x_{1}, x_{2}^{m}\right)$ and $\left(x_{1}^{n}, x_{2}\right)$ is adjacent to the vertex $\left(x_{1}^{n}, x_{2}^{m}\right)$. These facts can be shown systematically as

$$
\begin{aligned}
& \left(x_{1}, x_{2}\right)-\left(x_{1}, x_{2}^{m}\right)-\left(x_{1}^{n}, x_{2}^{m}\right)-\left(x_{1}^{i}, x_{2}^{m}\right)-\left(x_{1}^{i}, x_{2}^{j}\right) \text { and } \\
& \left(x_{1}, x_{2}\right)-\left(x_{1}^{n}, x_{2}\right)-\left(x_{1}^{n}, x_{2}^{m}\right)-\left(x_{1}^{n}, x_{2}^{j}\right)-\left(x_{1}^{i}, x_{2}^{j}\right) .
\end{aligned}
$$

Therefore, $\operatorname{diam}(\mathcal{T})=4$, as required.

\section{Theorem 11}

$$
\operatorname{girth}(\mathcal{T})=3
$$

Proof Since $x^{n} \cdot x^{n-1}=0, x^{n-1} \cdot x^{2}=0$ and $x^{n} \cdot x^{i}=0$, we clearly have $\left(x_{1}^{n}, x_{2}^{m}\right)-\left(x_{1}^{n}, x_{2}^{m-1}\right)-$ $\left(x_{1}^{n}, x_{2}^{2}\right)-\left(x_{1}^{n}, x_{2}^{m}\right)$. This gives the result.

We note that the chromatic number of the Cartesian product of simple graphs $G_{1}$ and $G_{2}$ satisfies the equality $\chi\left(G_{1} \square G_{2}\right)=\max \left\{\chi\left(G_{1}\right), \chi\left(G_{2}\right)\right\}$ (cf. [20]). Now, we replace $G_{1}$ by $\mathcal{S}_{M}^{1}$ and $G_{2}$ by $\mathcal{S}_{M}^{2}$, and then considering Theorem 6 , we clearly obtain the following result about the chromatic number for the graph $\mathcal{T}$.

\section{Theorem 12}

$$
\chi(\mathcal{T})=1+\left\lceil\frac{n-1}{2}\right\rceil
$$

Furthermore, we also get the next theorem for the clique number of $\mathcal{T}$.

\section{Theorem 13}

$$
\omega(\mathcal{T})=1+\left\lceil\frac{n-1}{2}\right\rceil
$$

Proof Now, let us consider the graph $\mathcal{T}$ with its subgraph $A$. In this case, for all distinct vertices $\left(x_{1}^{i}, x_{2}^{j}\right)$ and $\left(x_{1}^{a}, x_{2}^{b}\right)$, a subgraph will be definitely complete if

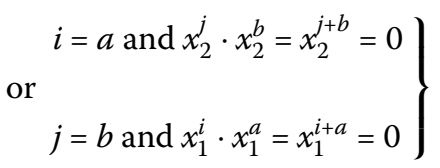

i.e., for all related powers $i, j, a$ and $b$, the subgraph will be complete if $\left(x_{1}^{i}, x_{2}^{j}\right)\left(x_{1}^{a}, x_{2}^{b}\right) \in$ $E(\mathcal{T})$.

On the other hand, each of the equalities in (8) will only be satisfied in case the sum $j+b$ is at least equal to $m+1$ and the sum $i+a$ is at least equal to $n+1$. By the assumption $n \geq m$, let us consider the case $j=b$. Therefore, by a similar idea to that in the proof of Theorem 7 , for any two vertices $x_{1}^{i}$ and $x_{1}^{a}$, we get

$$
i=\left\lceil\frac{n}{2}\right\rceil \text { and } \quad a=\left\lceil\frac{n}{2}\right\rceil+1
$$


In fact, these above determinations imply that $A \subseteq \mathcal{T}$ is a maximal complete subgraph with the vertex set

$$
V(A)=\left\{\left(x_{1}^{\left\lceil\frac{n}{2}\right\rceil}, x_{2}^{j}\right),\left(x^{\left\lceil\frac{n}{2}\right\rceil+1}, x_{2}^{j}\right), \ldots,\left(x^{n-1}, x_{2}^{j}\right),\left(x^{n}, x_{2}^{j}\right)\right\} .
$$

A simple calculation shows that

$$
\begin{aligned}
|V(A)| & =n-\left\lceil\frac{n}{2}\right\rceil+1 \\
& =\left\lceil\frac{n-1}{2}\right\rceil+1 \quad \text { by Lemma } 1 .
\end{aligned}
$$

Hence, we obtain $\omega(\mathcal{T})=1+\left\lceil\frac{n-1}{2}\right\rceil$ as required.

Remark 2 For any two graphs $G_{1}$ and $G_{2}$, it is presented in [17] that $\omega\left(G_{1} \square G_{2}\right) \geq$ $\max \left\{\omega\left(G_{1}\right), \omega\left(G_{2}\right)\right\}$. However, by considering Theorems 11 and 13, since $\chi(\mathcal{T})=\omega(\mathcal{T})=$ $1+\left\lceil\frac{n-1}{2}\right\rceil$, we obtain the strict equality $\omega(\mathcal{T})=\max \left\{\omega\left(\Gamma\left(\mathcal{S}_{M}^{1}\right)\right), \omega\left(\Gamma\left(\mathcal{S}_{M}^{2}\right)\right)\right\}$ for our special graphs studied in this paper.

\section{Competing interests}

The authors declare that they have no competing interests.

\section{Authors' contributions}

All authors completed the paper together. All authors read and approved the final manuscript.

\section{Author details}

'Department of Mathematics, Sungkyunkwan University, Suwon, 440-746, Republic of Korea. ${ }^{2}$ Department of Mathematics, Faculty of Science, Selçuk University, Campus, Konya, 42075, Turkey.

\section{Acknowledgements}

The first author is supported by the Faculty Research Fund, Sungkyunkwan University, 2012 and Sungkyunkwan University BK21 Project, BK21 Math Modelling HRD Div. Sungkyunkwan University, Suwon, Republic of Korea. The second and third authors are both partially supported by the Research Project Office of Selçuk University. Some of the material in this paper can also be found in the second author's Ph.D. thesis.

Received: 21 November 2012 Accepted: 18 January 2013 Published: 11 February 2013

\section{References}

1. Beck, I: Coloring of commutating ring. J. Algebra 116, 208-226 (1988)

2. Anderson, DF, Livingston, PS: The zero-divisor graph of commutative ring. J. Algebra 217, 434-447 (1999)

3. Anderson, DF, Badawi, A: On the zero-divisor graph of a ring. Commun. Algebra 36(8), 3073-3092 (2008)

4. Anderson, DD, Naseer, M: Beck's coloring of a commutative ring. J. Algebra 159, 500-514 (1991)

5. Akbari, S, Maimani, HR, Yassemi, S: When a zero-divisor graph is planar or a complete $r$-partite graph. J. Algebra 270, 169-180 (2003)

6. Akgunes, N, Togan, M: Some graph theoretical properties over zero-divisor graphs of special finite commutative rings. Adv. Stud. Contemp. Math. 22(2), 305-315 (2012)

7. DeMeyer, FR, DeMeyer, L: Zero-divisor graphs of semigroups. J. Algebra 283, 190-198 (2005)

8. DeMeyer, FR, McKenzie, T, Schneider, K: The zero-divisor graph of a commutative semigroup. Semigroup Forum 65 206-214 (2002)

9. Wright, SE: Lengths of paths and cycles in zero-divisor graphs and digraphs of semi-groups. Commun. Algebra 35, 1987-1991 (2007)

10. Gross, JL, Yellen, J: Handbook of Graph Theory. Chapman \& Hall/CRC, London (2004)

11. Mukwembi, S: A note on diameter and the degree sequence of a graph. Appl. Math. Lett. 25, 175-178 (2012)

12. Lovász, L: Normal hypergraphs and the weak perfect graph conjecture. Discrete Math. 2, 253-267 (1972)

13. Berge, C: The Theory of Graphs and Its Applications, x+247 pp. Wiley, New York (1962). Translated by Alison Doig Methuen \& Co. Ltd., London

14. Chudnovsky, M, Robertson, N, Seymour, P, Thomas, R: The strong perfect graph theorem. Ann. Math. 164, 51-229 (2006)

15. Bollobás, B: Modern Graph Theory. Graduate Texts in Mathematics, vol. 184. Springer, New York (1998)

16. Das, KC, Gutman, I: Estimating the Szeged index. Appl. Math. Lett. 22, 1680-1684 (2009) 
17. Doŝlić, T, Ghorbani, M, Hosseinzadeh, MA: The relationships between Wiener index, stability number and clique number of composite graphs. Bull. Malays. Math. Soc. 36(1), 165-172 (2013)

18. Vizing, VG: The Cartesian product of graphs. Vyčisl. Sist. 9, 30-43 (1963) (in Russian)

19. Xu, JM, Yang, C: Connectivity of Cartesian product graphs. Discrete Math. 306(1), 159-165 (2006)

20. Sabidussi, G: Graphs with given group and given graph-theoretical properties. Can. J. Math. 9, 515-525 (1957)

doi:10.1186/1029-242X-2013-44

Cite this article as: Das et al.: On a graph of monogenic semigroups. Journal of Inequalities and Applications 2013 2013:44.

Submit your manuscript to a SpringerOpen ${ }^{\circ}$ journal and benefit from:

- Convenient online submission

Rigorous peer review

- Immediate publication on acceptance

- Open access: articles freely available online

- High visibility within the field

- Retaining the copyright to your article 